\title{
NEW PRECISION ORBITS OF BRIGHT DOUBLE-LINED SPECTROSCOPIC BINARIES. V. THE AM STARS HD 434 AND 41 SEXTANTIS
}

\author{
Francis C. FeKel ${ }^{1}$ and Michael H. Williamson \\ Center of Excellence in Information Systems, Tennessee State University, 3500 John A. Merritt Boulevard, Box 9501, Nashville, TN 37209, USA; \\ fekel@evans.tsuniv.edu \\ Received 2010 August 6; accepted 2010 August 27; published 2010 October 14
}

\begin{abstract}
We have detected the secondary component in two previously known spectroscopic binaries, HD 434 and 41 Sex, and for the first time determined double-lined orbits for them. Despite the relatively long period of 34.26 days and a moderate eccentricity of 0.32 , combined with the components' rotationally broadened lines, measurement of the primary and secondary radial velocities of HD 434 has enabled us to obtain significantly improved orbital elements. While the 41 Sex system has a much shorter period of 6.167 days and a circular orbit, the estimated $V$ mag difference of 3.2 between its components also makes this a challenging system. The new orbital dimensions $\left(a_{1} \sin i\right.$ and $\left.a_{2} \sin i\right)$ and minimum masses $\left(m_{1} \sin ^{3} i\right.$ and $\left.m_{2} \sin ^{3} i\right)$ of HD 434 have accuracies of $0.8 \%$ or better, while the same quantities for 41 Sex are good to $0.5 \%$ or better. Both components of HD 434 are Am stars while the Am star primary of 41 Sex has a late-F or early-G companion. All four stars are on the main sequence. The two components of HD 434 are rotating much faster than their predicted pseudosynchronous velocities, while both components of 41 Sex are synchronously rotating. For the primary of $41 \mathrm{Sex}$, the spectrum line depth changes noted by Sreedhar Rao et al. were not detected.
\end{abstract}

Key words: binaries: spectroscopic - stars: individual (HD 434, 41 Sex)

\section{INTRODUCTION}

Direct determination of stellar masses and precise stellar parallaxes can be obtained from the three-dimensional orbits of binary stars that are resolved as both a spectroscopic and a visual binary. During the past two decades, major improvements in ground-based optical and near-infrared interferometry have produced a greater overlap of spectroscopic and visual binary domains (Quirrenbach 2001). Cunha et al. (2007) provided a list of over 30 interferometric visual orbits for double-lined spectroscopic binaries, while Torres et al. (2010) identified 23 interferometric systems with stellar masses determined to better than 3\%. Work on individual systems (e.g., Hummel et al. 2001; Boden et al. 2006; Fekel et al. 2009a) has led to useful comparisons with evolutionary theory.

In SB9, the continually updated Web-based edition of the spectroscopic binary orbit catalog (Pourbaix et al. 2004), many of the older spectroscopic orbits have been computed with radial velocities from photographic plates. Because the photographic plates generally have lower resolutions and lower signal-tonoise ratios than modern CCD observations, results from them limit the precision of three-dimensional orbits. Thus, in previous papers of this series (Tomkin \& Fekel 2006, 2008; Fekel et al. 2009 b, 2010) we have obtained new radial velocities and computed spectroscopic orbits for bright field spectroscopic binaries, which are the most accessible systems to interferometry. These improved spectroscopic orbits will greatly enhance prospective interferometric observations of these systems.

To obtain the component masses and parallax of a binary system, spectroscopic orbits of the two components are needed. Although HD 434 and 41 Sex have only single-lined orbits in the literature, at red wavelengths the secondary components of

\footnotetext{
1 Visiting Astronomer, Kitt Peak National Observatory, National Optical Astronomy Observatory, operated by the Association of Universities for Research in Astronomy, Inc. under cooperative agreement with the National Science Foundation.
}

both spectroscopic binaries are visible, turning the two systems into viable candidates for three-dimensional orbital solutions.

The spectroscopic binaries, HD 434 and 41 Sex, that are analyzed in this paper have Am star primaries. Sreedhar Rao \& Abhyankar (1992) previously examined the two systems together, revising their orbits and discussing the stars. Table 1 gives some of their basic properties.

\section{BRIEF HISTORY}

$$
\text { 2.1. HD } 434=H I P 728=S A O 73772
$$

HD $434\left(\alpha=00^{\mathrm{h}} 09^{\mathrm{m}} 00^{\mathrm{s}} .16, \delta=28^{\circ} 14^{\prime} 51^{\prime \prime} \cdot 2\right.$ (2000)) was not included in the Bright Star Catalogue (Hoffleit 1982), which has a nominal magnitude limit of $V=6.5$. However, in Appendix III of that catalog it is noted as one of a number of stars that modern photoelectric photometry has shown to be slightly brighter than the catalog limiting magnitude (Hoffleit 1982). Interest in HD 434 came slowly. Shajn (1951) discovered that it had radial velocity variations and classified its spectrum as A2s. However, Walker (1966) identified it as an Am star. Palmer et al. (1968) observed HD 434 as part of a spectroscopic survey of 633 bright, northern A stars and confirmed its velocity variations. Eventually, Hube \& Gulliver (1985) obtained over three dozen additional radial velocities from which they computed a preliminary single-lined orbit with a period of 34.2601 days. Both Sreedhar Rao \& Abhyankar (1992) and Margoni et al. (1992) collected additional observations, and using previous velocity observations, the two groups independently revised the preliminary orbit of Hube \& Gulliver (1985). As part of an extended project on Am binaries, Iliev et al. (2001) reobserved HD 434 and announced the discovery of its secondary spectrum. From those spectra, Budaj et al. (2003) obtained a mass ratio of 1.19 for the primary relative to the secondary.

Walker (1966) used the strength of the $\mathrm{Ca} \mathrm{K}$ line relative to the Balmer lines to estimate a spectral class of A4, which was repeated by Palmer et al. (1968). Bertaud (1970) classified 
Table 1

Basic Properties of the Program Stars

\begin{tabular}{rrrccrrr}
\hline \hline Name & HR & HD & Spectral Type & $V^{\mathrm{a}}$ & $B-V^{\mathrm{a}}$ & $\begin{array}{r}\text { Parallax }^{\mathrm{b}} \\
(\mathrm{mas})\end{array}$ & $\begin{array}{r}\text { Period } \\
(\text { days })\end{array}$ \\
\hline$\ldots$ & $\ldots$ & 434 & Am & 6.47 & 0.242 & 9.02 & 34.26 \\
$41 \mathrm{Sex}$ & 4237 & 93903 & Am & 5.80 & 0.156 & 10.50 & 6.17 \\
\hline
\end{tabular}

Notes.

${ }^{\text {a }}$ Perryman et al. (1997).

b van Leeuwen (2007).

the $\mathrm{Ca} \mathrm{K}$ and metal lines as $\mathrm{A} 3$ and $\mathrm{F} 0$, respectively. More recently Abt (2004) also identified the combined spectrum as that of a classical Am star and provided spectral classes for its $\mathrm{Ca} \mathrm{K}$, hydrogen, and metal lines of A2, A6, and F0, respectively. Examining their red wavelength spectra where both components of the binary were in evidence, Iliev et al. (2001) concluded that both stars have Am peculiarities.

Walker (1966) estimated a $v \sin i$ value of $60 \pm 25 \mathrm{~km} \mathrm{~s}^{-1}$. However, the significantly higher resolution observations of Iliev et al. (2001) resulted in values of about 32 and $27 \mathrm{~km} \mathrm{~s}^{-1}$ for the primary and secondary, respectively.

\subsection{Sex $=H R 4237=H D 93903$}

Cowley et al. (1969) included $41 \mathrm{Sex}\left(\alpha=10^{\mathrm{h}} 50^{\mathrm{m}} 18 \mathrm{~s} .06\right.$, $\delta=-08^{\circ} 53^{\prime} 51^{\prime \prime} .9$ (2000)) in a study of the spectral classifications of bright A stars. They characterized its spectrum as that of an Am star and found its $\mathrm{Ca} \mathrm{K}$ line to have an A3 spectral class. Because most Am stars are binaries (Abt 1961; Abt \& Levy 1985), Worek et al. (1978) obtained radial velocities of the system and, as expected, found it to be a spectroscopic binary. They determined an orbital period of 6.1670 days and concluded that it had a nearly circular orbit. Abt \& Levy (1985) made 41 Sex part of their improved study of Am stars. From their spectroscopic observations they computed a new orbit that confirmed the results of Worek et al. (1978). Shortly thereafter, Worek et al. (1986) also published an improved solution of 41 Sex, which generally agreed with the previous two. Over the next dozen years, there were two additional attempts to upgrade the orbital solution of 41 Sex. Sreedhar Rao \& Abhyankar (1992) combined all previously published velocities with an extensive number of newly obtained measurements to produce another revised orbit. Meanwhile, Worek (1998) continued his work on 41 Sex, computing the most recent revision of its orbit.

After the initial recognition of 41 Sex as an Am star by Cowley et al. (1969), Abt \& Levy (1985) in their improved study of Am binaries classified the star as A2, A8, and F0 from its $\mathrm{Ca} \mathrm{K}$, hydrogen, and metal lines, respectively. Later, Abt \& Morrell (1995) included 41 Sex in an extensive survey of A stars that examined the relationship between rotational velocity and spectral peculiarities. From their new analysis they estimated spectral classes of A3, A7, and A9, very similar to the results of Abt \& Levy (1985). They also measured a $v \sin i$ value of $18 \mathrm{~km} \mathrm{~s}^{-1}$ for the primary. Royer et al. (2002) rescaled that result to $26 \mathrm{~km} \mathrm{~s}^{-1}$, while Fekel (2003) obtained a value of $21 \mathrm{~km} \mathrm{~s}^{-1}$.

From moderate dispersion photographic plates, Sreedhar Rao et al. (1990) announced the discovery of phase-modulated variations of the $\mathrm{Ca} \mathrm{K}$, hydrogen lines, and various metal lines in the spectrum of 41 Sex. They interpreted their results as surface abundance inhomogenities similar to the $\mathrm{Sr}, \mathrm{Cr}$, and $\mathrm{Eu}$ line enhancements found in Ap stars and suggested that the characteristics of the abundance peculiarities linked the

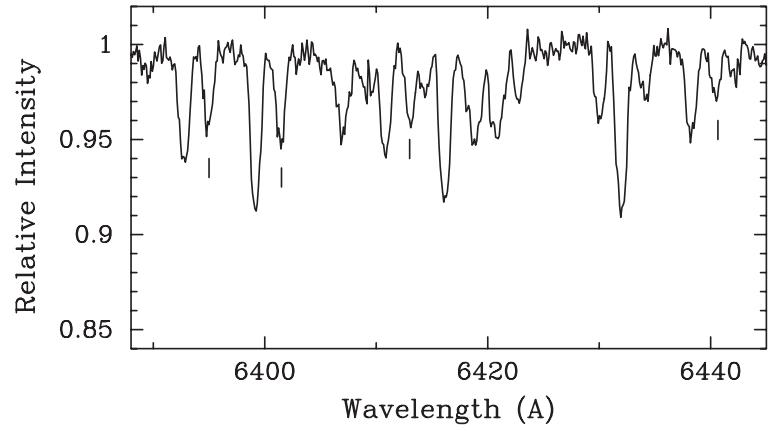

Figure 1. Spectrum of HD 434 in the 6430 Å region. Tick marks identify several redshifted secondary components.

heretofore separate Am and Ap stars. Although Sreedhar Rao \& Abhyankar (1992) concluded that 41 Sex is a prototype for a new class of spectrum variables among Am stars, they did caution that higher resolution CCD spectra would be useful to confirm the spectral line variations. Worek (1998) took up their challenge and obtained CCD spectra of 41 Sex. His careful analysis of the new spectra did not confirm the line variability claims of Sreedhar Rao et al. (1990).

\section{OBSERVATIONS AND RADIAL VELOCITIES}

In 2005, a lone double-lined spectrum of HD 434 was acquired at McDonald Observatory with the $2.1 \mathrm{~m}$ telescope, the Sandiford Cassegrain echelle spectrograph (McCarthy et al. 1993), and a Reticon CCD. The spectrogram covers the wavelength range 5700-7000 $\AA$ and has a resolving power of 49,000.

From 2001 through 2010 we obtained spectrograms of both HD 434 and 41 Sex at the Kitt Peak National Observatory (KPNO) with the coudé feed telescope and coudé spectrograph. All observations were made with a TI CCD detector, and the spectra are centered at $6430 \AA$, cover a wavelength range of $84 \AA$, and have a resolution of $0.21 \AA$ or a resolving power of just over 30,000. Further details about our observations and data reduction are given in Tomkin \& Fekel (2006).

Finally, from 2003 through 2010 we collected an extensive number of observations of the two systems with the Tennessee State University $2 \mathrm{~m}$ Automatic Spectroscopic Telescope (AST), a fiber-fed echelle spectrograph, and a 2048×4096 SITe ST-002A CCD. The echelle spectrograms have 21 orders that cover the wavelength range 4920-7100 $\AA$ with an average resolution of $0.17 \AA$. The typical signal-to-noise ratio of these observations is $\sim 80$. Eaton \& Williamson $(2004,2007)$ have given a more extensive description of the telescope and spectrograph, operated at Fairborn Observatory near Washington Camp in the Patagonia Mountains of southeastern Arizona.

The spectra of the two systems show both components. However, for HD 434 the broad lines and low velocity amplitudes of the stars result in the lines of the two components being blended throughout much of the orbit. Fortunately, the components are completely separated at phases near periastron, as seen in Figure 1. For 41 Sex, the secondary lines are completely separated from their primary counterparts (Figure 2) at nearly all phases.

The procedures used to measure the McDonald and KPNO radial velocities have been described extensively in Tomkin \& Fekel (2006). Here, we note that the McDonald velocities are absolute velocities, which were placed on a secure rest scale by means of the telluric $\mathrm{O}_{2}$ and $\mathrm{H}_{2} \mathrm{O}$ lines in the stellar spectra. The KPNO velocities were determined by cross-correlation with 


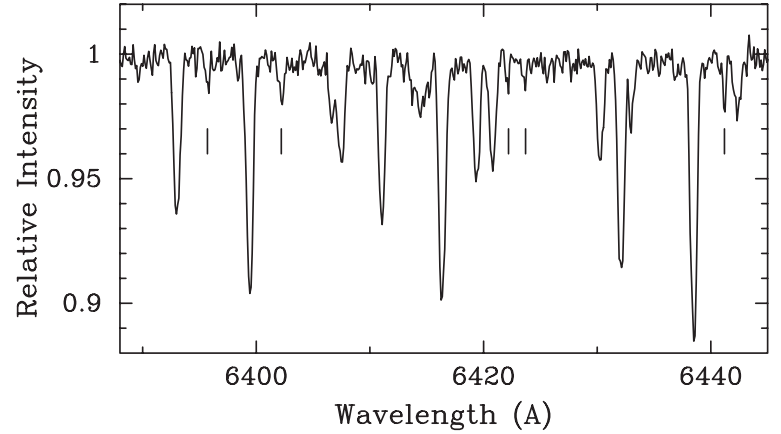

Figure 2. Spectrum of 41 Sex in the $6430 \AA$ A region. Tick marks identify several redshifted secondary components.

respect to IAU radial velocity standard stars of the same or similar spectral type as the program stars. The velocities adopted for those standards are from Scarfe et al. (1990).

Fekel et al. (2009b) provided an extensive general description of velocity measurement of the Fairborn AST spectra. Here, we mention that for HD 434 and 41 Sex the lines that were chosen for measurement were determined by the spectral classes of the stars. The component lines of the Am star HD 434 are roughly similar in strength, and the mean spectral class of the metal lines is F0 (Abt 2004). Thus, we used a line list that consists mostly of singly ionized elements such as Fe II, Si II, Ti II, and Cr II, which are prominent features in A and early-F stars. The primary of $41 \mathrm{Sex}$ is also an Am star that has metal lines with a spectral class of about F0 (Abt \& Levy 1985). However, to enhance our detection of the lines of the much cooler secondary, for both components we used a line list that consists mostly of Fe I lines, which is used for stars with spectral types of early-F through M, rather than the line list for A stars. The resulting Fairborn velocities, like those from McDonald Observatory, are absolute velocities. Our unpublished velocities of several IAU standard solar-type stars indicate that the Fairborn Observatory velocities have a small zero-point offset of $-0.3 \mathrm{~km} \mathrm{~s}^{-1}$ relative to the velocities of Scarfe et al. (1990). Thus, we have added $0.3 \mathrm{~km} \mathrm{~s}^{-1}$ to each Fairborn velocity.

\section{DETERMINATION OF ORBITS AND RESULTS}

We have used several computer programs to determine the orbital elements. Preliminary orbits were computed with the program BISP (Wolfe et al. 1967), which implements a slightly modified version of the Wilsing-Russell method. Eccentric orbits were then determined with SB1 (Barker et al. 1967), a program that uses differential corrections. For a simultaneous solution of the two components of HD 434, we used SB2, which is a slightly modified version of SB1. For 41 Sex, circular orbits were determined with SB1C and SB2C (D. Barlow 1998, private communication), which also use differential corrections to compute improved orbital elements.

The spectra that we obtained at the different observatories have different wavelength ranges. Thus, different numbers of lines were available for measurement. In addition, the primary and secondary lines are of different quality because the components differ in strength and in line width. Thus, the velocity precision of each set of data will vary from observatory to observatory, and the precision will also usually differ for each primary and secondary component. To determine the weight for each set of our velocities, as well as those from the literature, we computed the variances of the individual orbital solutions, which are inversely proportional to our adopted weights.

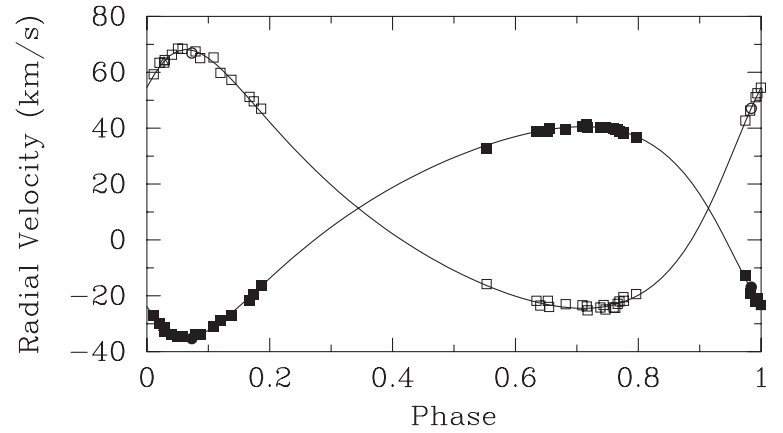

Figure 3. Radial velocities of HD 434 compared with the computed velocity curves. Filled and open symbols represent the primary and secondary, respectively. Squares, Fairborn Observatory; circles, KPNO; triangles, McDonald Observatory. Zero phase is a time of periastron passage.

\section{1. $H D 434$}

For HD 434 the line broadening and modest velocity amplitudes of its components, combined with its roughly 1 month orbital period, caused us to obtain the vast majority of our observations at Fairborn Observatory, where from 2003 through 2010 we acquired 39 spectra that had resolved or mostly resolved spectral lines of the two components. At McDonald Observatory and $\mathrm{KPNO}$ we collected only three additional double-lined spectra, one from McDonald and two from KPNO. All our velocities are listed in Table 2. Using the Fairborn velocities for the two components, we computed single-lined orbital solutions, which resulted in weights of 1.0 and 0.5 for the Fairborn primary and secondary velocities, respectively. Because of their small number, we simply adopted the same weights for the primary and secondary velocities of our three McDonald and KPNO observations. A simultaneous solution of the weighted primary and secondary velocities produced a period of 34.2624 days.

The spectral resolution of the various earlier photographic plate data sets was not sufficient to separate the components. Thus, the earlier velocity measurements are blends of the primary and secondary, and so we have not attempted to use those velocities to improve the orbit. Table 3 lists the orbital elements from our simultaneous solution of the two components with only our data. Figure 3 compares our primary and secondary velocities with the calculated velocity curves. Zero phase is a time of periastron passage.

In addition to our new elements, Table 3 lists the preliminary elements of Hube \& Gulliver (1985), and there are significant differences between the two solutions. Our center-of-mass velocity is more than $4 \mathrm{~km} \mathrm{~s}^{-1}$ greater than that of Hube \& Gulliver (1985), which is probably mostly due to their measurement of blended lines. In addition, our eccentricity is $20 \%$ smaller than that of Hube \& Gulliver (1985), while our semiamplitude of the primary is $6 \%$ larger.

\section{2. $41 \mathrm{Sex}$}

For 41 Sex we acquired 34 observations at KPNO and 49 at Fairborn Observatory between 2001 and 2010 (Table 4). After measuring the primary and secondary velocities we computed four independent single-lined orbital solutions for the four data sets. Comparing the variances of those solutions, the KPNO velocities are the most precise, and we adopted weights of 1.0, 0.3, 0.04, and 0.01 for the KPNO primary, Fairborn primary, KPNO secondary, and Fairborn secondary velocities, respectively. The center-of-mass velocities of the four data sets are in good agreement except for that of the KPNO secondary. 
Table 2

Radial Velocities of HD 434

\begin{tabular}{|c|c|c|c|c|c|c|c|c|}
\hline $\begin{array}{l}\text { Heliocentric Julian Date } \\
\text { (HJD - 2,400,000) }\end{array}$ & Phase & $\begin{array}{c}V_{1} \\
\left(\mathrm{~km} \mathrm{~s}^{-1}\right)\end{array}$ & $\begin{array}{c}(O-C)_{1} \\
\left(\mathrm{~km} \mathrm{~s}^{-1}\right)\end{array}$ & $\mathrm{Wt}_{1}$ & $\begin{array}{c}V_{2} \\
\left(\mathrm{~km} \mathrm{~s}^{-1}\right)\end{array}$ & $\begin{array}{c}(O-C)_{2} \\
\left(\mathrm{~km} \mathrm{~s}^{-1}\right)\end{array}$ & $\mathrm{Wt}_{2}$ & Source ${ }^{\mathrm{a}}$ \\
\hline 52966.736 & 0.716 & 41.2 & 0.7 & 1.0 & -23.8 & 0.7 & 0.5 & Fair \\
\hline 52967.675 & 0.744 & 40.3 & 0.2 & 1.0 & -23.3 & 0.7 & 0.5 & Fair \\
\hline 53032.665 & 0.640 & 39.0 & 0.0 & 1.0 & -23.5 & -0.9 & 0.5 & Fair \\
\hline 53276.818 & 0.766 & 39.6 & 0.4 & 1.0 & -22.9 & -0.1 & 0.5 & Fair \\
\hline 53285.789 & 0.028 & -32.7 & -1.1 & 1.0 & 63.5 & -0.5 & 0.5 & Fair \\
\hline 53290.782 & 0.174 & -19.7 & -0.7 & 1.0 & 49.6 & 1.0 & 0.5 & Fair \\
\hline 53303.772 & 0.553 & 32.8 & -1.1 & 1.0 & -15.8 & 0.5 & 0.5 & Fair \\
\hline 53310.852 & 0.760 & 39.8 & 0.3 & 1.0 & -24.2 & -1.0 & 0.5 & Fair \\
\hline 53318.878 & 0.994 & -21.0 & 0.8 & 1.0 & 52.5 & 0.5 & 0.5 & Fair \\
\hline 53340.810 & 0.634 & 38.9 & 0.2 & 1.0 & -21.8 & 0.5 & 0.5 & Fair \\
\hline 53352.730 & 0.982 & -19.1 & -2.0 & 1.0 & 46.3 & 0.0 & 0.5 & Fair \\
\hline 53353.726 & 0.011 & -27.1 & 0.3 & 1.0 & 59.3 & 0.4 & 0.5 & Fair \\
\hline 53359.718 & 0.186 & -16.5 & 0.1 & 1.0 & 47.0 & 1.4 & 0.5 & Fair \\
\hline 53649.879 & 0.655 & 39.9 & 0.4 & 1.0 & -24.0 & -0.7 & 0.5 & Fair \\
\hline 53662.667 & 0.028 & -31.3 & 0.2 & 1.0 & 63.6 & -0.4 & 0.5 & $\mathrm{McD}$ \\
\hline 53687.836 & 0.763 & 39.4 & 0.0 & 1.0 & -24.3 & -1.2 & 0.5 & Fair \\
\hline 54003.775 & 0.984 & -16.9 & 0.9 & 1.0 & 47.1 & 0.0 & 0.5 & KPNO \\
\hline 54006.834 & 0.073 & -35.3 & -0.6 & 1.0 & 66.9 & -1.0 & 0.5 & KPNO \\
\hline 54860.584 & 0.991 & -22.0 & -1.4 & 1.0 & 51.1 & 0.5 & 0.5 & Fair \\
\hline 54861.589 & 0.020 & -30.1 & -0.2 & 1.0 & 63.4 & 1.5 & 0.5 & Fair \\
\hline 54862.628 & 0.051 & -34.4 & 0.1 & 1.0 & 68.5 & 0.9 & 0.5 & Fair \\
\hline 54863.605 & 0.079 & -33.7 & 0.7 & 1.0 & 67.5 & -0.1 & 0.5 & Fair \\
\hline 54864.610 & 0.109 & -31.1 & 0.1 & 1.0 & 65.3 & 1.7 & 0.5 & Fair \\
\hline 54865.606 & 0.138 & -26.9 & -0.7 & 1.0 & 57.3 & -0.2 & 0.5 & Fair \\
\hline 54866.613 & 0.167 & -21.7 & -1.3 & 1.0 & 51.2 & 0.8 & 0.5 & Fair \\
\hline 55159.574 & 0.718 & 40.4 & -0.1 & 1.0 & -25.2 & -0.7 & 0.5 & Fair \\
\hline 55160.573 & 0.747 & 40.1 & 0.1 & 1.0 & -24.9 & -1.1 & 0.5 & Fair \\
\hline 55161.572 & 0.776 & 38.3 & -0.4 & 1.0 & -20.5 & 1.7 & 0.5 & Fair \\
\hline 55332.910 & 0.777 & 38.2 & -0.4 & 1.0 & -21.8 & 0.3 & 0.5 & Fair \\
\hline 55341.958 & 0.041 & -33.8 & -0.3 & 1.0 & 66.3 & -0.1 & 0.5 & Fair \\
\hline 55362.927 & 0.653 & 38.8 & -0.7 & 1.0 & -21.8 & 1.4 & 0.5 & Fair \\
\hline 55363.927 & 0.682 & 39.4 & -0.9 & 1.0 & -23.0 & 1.1 & 0.5 & Fair \\
\hline 55364.860 & 0.709 & 40.5 & 0.0 & 1.0 & -23.4 & 1.1 & 0.5 & Fair \\
\hline 55365.860 & 0.738 & 40.4 & 0.2 & 1.0 & -24.2 & -0.1 & 0.5 & Fair \\
\hline 55366.928 & 0.769 & 38.7 & -0.3 & 1.0 & -22.0 & 0.6 & 0.5 & Fair \\
\hline 55367.860 & 0.797 & 36.7 & -0.3 & 1.0 & -19.4 & 0.8 & 0.5 & Fair \\
\hline 55373.948 & 0.974 & -12.6 & 1.3 & 1.0 & 42.7 & 0.4 & 0.5 & Fair \\
\hline 55374.818 & 1.000 & -23.3 & 0.5 & 1.0 & 54.5 & 0.0 & 0.5 & Fair \\
\hline 55375.818 & 0.029 & -31.2 & 0.5 & 1.0 & 64.4 & 0.2 & 0.5 & Fair \\
\hline 55376.818 & 0.058 & -34.5 & 0.3 & 1.0 & 68.4 & 0.3 & 0.5 & Fair \\
\hline 55377.798 & 0.087 & -33.7 & 0.2 & 1.0 & 65.1 & -1.7 & 0.5 & Fair \\
\hline 55378.929 & 0.120 & -28.9 & 0.6 & 1.0 & 59.8 & -1.6 & 0.5 & Fair \\
\hline
\end{tabular}

Note. ${ }^{\text {a }}$ Fair, Fairborn Observatory; KPNO, Kitt Peak National Observatory; McD, McDonald Observatory.

Table 3

Orbital Elements and Related Parameters of HD 434

\begin{tabular}{lcc}
\hline \multicolumn{1}{c}{ Parameter } & Hube \& Gulliver (1985) & This Study \\
\hline$P$ (days) & 34.26014 & $34.26241 \pm 0.00061$ \\
$T$ (HJD) & $2443041.27 \pm 0.42$ & $2454175.643 \pm 0.043$ \\
$e$ & $0.405 \pm 0.033$ & $0.3200 \pm 0.0038$ \\
$\omega_{1}(\mathrm{deg})$ & $140.9 \pm 6.7$ & $135.01 \pm 0.55$ \\
$K_{1}\left(\mathrm{~km} \mathrm{~s}^{-1}\right)$ & $35.5 \pm 1.7$ & $37.74 \pm 0.12$ \\
$K_{2}\left(\mathrm{~km} \mathrm{~s}^{-1}\right)$ & $\ldots$ & $46.33 \pm 0.17$ \\
$\gamma\left(\mathrm{km} \mathrm{s}^{-1}\right)$ & $6.9 \pm 0.7$ & $11.351 \pm 0.093$ \\
$m_{1} \sin ^{3} i\left(M_{\odot}\right)$ & $\ldots$ & $0.9907 \pm 0.0084$ \\
$m_{2} \sin ^{3} i\left(M_{\odot}\right)$ & $\ldots$ & $0.8071 \pm 0.0063$ \\
$a_{1} \sin i\left(10^{6} \mathrm{~km}\right)$ & $15.30 \pm 0.76$ & $16.846 \pm 0.059$ \\
$a_{2} \sin i\left(10^{6} \mathrm{~km}\right)$ & $\ldots$ & $20.680 \pm 0.080$ \\
rms residual $\left(\mathrm{km} \mathrm{s}{ }^{-1}\right)($ unit weight) & $\ldots$ & 0.7 \\
\hline
\end{tabular}


No. 5, 2010

Table 4

Radial Velocities of 41 Sex

\begin{tabular}{|c|c|c|c|c|c|c|c|c|}
\hline $\begin{array}{l}\text { Heliocentric Julian Date } \\
\text { (HJD }-2,400,000)\end{array}$ & Phase & $\begin{array}{c}V_{1} \\
\left(\mathrm{~km} \mathrm{~s}^{-1}\right) \\
\end{array}$ & $\begin{array}{c}(O-C)_{1} \\
\left(\mathrm{~km} \mathrm{~s}^{-1}\right)\end{array}$ & $\mathrm{Wt}_{1}$ & $\begin{array}{c}V_{2} \\
\left(\mathrm{~km} \mathrm{~s}^{-1}\right)\end{array}$ & $\begin{array}{c}(O-C)_{2} \\
\left(\mathrm{~km} \mathrm{~s}^{-1}\right)\end{array}$ & $\mathrm{Wt}_{2}$ & Source $^{a}$ \\
\hline 52013.736 & 0.068 & 37.0 & 0.2 & 1.0 & -89.9 & 0.4 & 0.04 & KPNO \\
\hline 52015.737 & 0.393 & -42.1 & 0.1 & 1.0 & 68.8 & 1.8 & 0.04 & KPNO \\
\hline 52326.858 & 0.842 & 19.9 & 0.1 & 1.0 & -53.4 & 3.1 & 0.04 & KPNO \\
\hline 52327.851 & 0.003 & 40.9 & -0.1 & 1.0 & -98.9 & -0.2 & 0.04 & KPNO \\
\hline 52330.892 & 0.496 & -52.3 & 0.0 & 1.0 & 86.6 & -0.7 & 0.04 & KPNO \\
\hline 52392.658 & 0.512 & -52.4 & -0.2 & 1.0 & 86.2 & -0.9 & 0.04 & KPNO \\
\hline 52395.697 & 0.004 & 40.8 & -0.2 & 1.0 & -100.8 & -2.1 & 0.04 & KPNO \\
\hline 52706.836 & 0.456 & -50.6 & 0.0 & 1.0 & 84.3 & 0.4 & 0.04 & KPNO \\
\hline 52707.818 & 0.616 & -40.3 & 0.3 & 1.0 & 66.2 & 2.3 & 0.04 & KPNO \\
\hline 52755.730 & 0.385 & -40.4 & 0.2 & 1.0 & 65.2 & 1.2 & 0.04 & KPNO \\
\hline 52756.708 & 0.543 & -50.4 & 0.2 & 1.0 & 85.8 & 1.9 & 0.04 & KPNO \\
\hline 52758.723 & 0.870 & 26.2 & -0.1 & 1.0 & -68.2 & 1.2 & 0.04 & KPNO \\
\hline 53022.954 & 0.716 & -15.4 & 0.2 & 0.3 & 12.7 & -1.4 & 0.01 & Fair \\
\hline 53023.961 & 0.879 & 27.8 & -0.4 & 0.3 & -74.9 & -1.7 & 0.01 & Fair \\
\hline 53051.903 & 0.410 & -45.7 & -0.6 & 0.3 & 71.6 & -1.3 & 0.01 & Fair \\
\hline 53119.753 & 0.412 & -45.5 & -0.1 & 1.0 & 74.5 & 1.0 & 0.04 & KPNO \\
\hline 53120.719 & 0.569 & -47.8 & 0.3 & 1.0 & 78.0 & -0.8 & 0.04 & KPNO \\
\hline 53139.699 & 0.646 & -34.0 & 0.0 & 0.3 & 51.7 & 1.0 & 0.01 & Fair \\
\hline 53167.674 & 0.183 & 12.8 & -0.7 & 0.3 & -44.8 & -0.9 & 0.01 & Fair \\
\hline 53169.642 & 0.502 & -52.3 & 0.1 & 1.0 & 89.0 & 1.6 & 0.04 & KPNO \\
\hline 53172.641 & 0.988 & 40.8 & -0.1 & 1.0 & -98.4 & 0.1 & 0.04 & KPNO \\
\hline 53288.020 & 0.697 & -20.8 & 0.1 & 0.3 & 24.8 & 0.1 & 0.01 & Fair \\
\hline 53313.953 & 0.902 & 32.3 & -0.2 & 0.3 & -84.3 & -2.6 & 0.01 & Fair \\
\hline 53323.000 & 0.369 & -38.3 & -0.8 & 0.3 & 56.1 & -1.6 & 0.01 & Fair \\
\hline 53333.894 & 0.136 & 24.8 & -0.2 & 0.3 & -66.2 & 0.7 & 0.01 & Fair \\
\hline 53352.022 & 0.075 & 35.8 & -0.1 & 0.3 & -89.3 & -0.8 & 0.01 & Fair \\
\hline 53358.856 & 0.183 & 13.7 & 0.4 & 0.3 & -45.7 & -2.2 & 0.01 & Fair \\
\hline 53367.998 & 0.666 & -29.9 & -0.7 & 0.3 & 42.2 & 0.9 & 0.01 & Fair \\
\hline 53372.000 & 0.315 & -23.5 & 0.6 & 0.3 & 31.7 & 0.6 & 0.01 & Fair \\
\hline 53401.032 & 0.022 & 40.7 & 0.2 & 0.3 & -96.0 & 1.8 & 0.01 & Fair \\
\hline 53425.890 & 0.053 & 38.5 & 0.1 & 0.3 & -91.5 & 2.1 & 0.01 & Fair \\
\hline 53426.910 & 0.219 & 3.1 & -0.4 & 0.3 & -22.7 & 1.3 & 0.01 & Fair \\
\hline 53439.848 & 0.316 & -25.1 & -0.5 & 0.3 & 32.6 & 0.5 & 0.01 & Fair \\
\hline 53452.781 & 0.414 & -45.9 & -0.3 & 0.3 & 74.4 & 0.4 & 0.01 & Fair \\
\hline 53465.745 & 0.516 & -52.3 & -0.2 & 0.3 & 89.7 & 2.8 & 0.01 & Fair \\
\hline 53478.776 & 0.629 & -38.2 & -0.3 & 0.3 & 57.5 & -1.1 & 0.01 & Fair \\
\hline 53487.764 & 0.086 & 34.0 & -0.3 & 1.0 & -84.6 & 0.8 & 0.04 & KPNO \\
\hline 53490.753 & 0.571 & -48.0 & -0.2 & 1.0 & 77.3 & -1.0 & 0.04 & KPNO \\
\hline 53492.728 & 0.891 & 30.2 & -0.3 & 1.0 & -74.7 & 3.1 & 0.04 & KPNO \\
\hline 53504.681 & 0.829 & 17.0 & 0.4 & 0.3 & -49.2 & 1.0 & 0.01 & Fair \\
\hline 53527.681 & 0.559 & -49.1 & 0.1 & 0.3 & 80.3 & -0.8 & 0.01 & Fair \\
\hline 53534.634 & 0.686 & -23.5 & 0.4 & 1.0 & 31.1 & 0.5 & 0.04 & KPNO \\
\hline 53535.633 & 0.848 & 21.5 & 0.2 & 1.0 & -56.4 & 3.2 & 0.04 & KPNO \\
\hline 53536.634 & 0.011 & 40.8 & -0.1 & 1.0 & -98.6 & -0.1 & 0.04 & KPNO \\
\hline 53724.031 & 0.398 & -43.1 & -0.1 & 0.3 & 66.5 & -2.3 & 0.01 & Fair \\
\hline 53737.010 & 0.502 & -52.5 & -0.1 & 0.3 & 85.3 & -2.1 & 0.01 & Fair \\
\hline 53763.933 & 0.868 & 25.6 & -0.2 & 0.3 & -64.8 & 3.6 & 0.01 & Fair \\
\hline 53850.735 & 0.943 & 38.2 & 0.2 & 1.0 & -91.7 & 1.1 & 0.04 & KPNO \\
\hline 53851.728 & 0.104 & 31.4 & 0.0 & 1.0 & -81.6 & -2.0 & 0.04 & KPNO \\
\hline 53856.706 & 0.911 & 33.6 & -0.3 & 1.0 & -84.8 & -0.2 & 0.04 & KPNO \\
\hline 54220.697 & 0.933 & 36.9 & -0.1 & 1.0 & -91.4 & -0.7 & 0.04 & KPNO \\
\hline 54264.652 & 0.061 & 38.0 & 0.4 & 1.0 & -92.7 & -0.7 & 0.04 & KPNO \\
\hline 54526.782 & 0.566 & -48.6 & -0.2 & 1.0 & 78.3 & -1.2 & 0.04 & KPNO \\
\hline 54582.774 & 0.645 & -33.9 & 0.3 & 1.0 & 52.9 & 1.6 & 0.04 & KPNO \\
\hline 54849.905 & 0.961 & 39.4 & -0.2 & 0.3 & -92.0 & 4.0 & 0.01 & Fair \\
\hline 54898.842 & 0.897 & 31.5 & 0.0 & 0.3 & -80.7 & -0.9 & 0.01 & Fair \\
\hline 54905.905 & 0.042 & 39.2 & -0.2 & 0.3 & -95.6 & -0.1 & 0.01 & Fair \\
\hline 54918.850 & 0.141 & 23.0 & -0.9 & 0.3 & -66.2 & -1.6 & 0.01 & Fair \\
\hline 54946.713 & 0.659 & -31.1 & -0.2 & 1.0 & 46.0 & 1.3 & 0.04 & KPNO \\
\hline 54948.713 & 0.983 & 41.0 & 0.3 & 0.3 & -94.1 & 4.1 & 0.01 & Fair \\
\hline $54982.735^{\mathrm{b}}$ & 0.500 & -53.3 & -0.9 & 0.3 & $\ldots$ & $\ldots$ & $\ldots$ & Fair \\
\hline 55006.636 & 0.376 & -38.6 & 0.2 & 1.0 & 62.5 & 2.1 & 0.04 & KPNO \\
\hline 55159.008 & 0.083 & 34.4 & -0.3 & 0.3 & -86.7 & -0.4 & 0.01 & Fair \\
\hline $55234.899^{\mathrm{b}}$ & 0.389 & -41.9 & -0.4 & 0.3 & $\ldots$ & $\ldots$ & $\ldots$ & Fair \\
\hline 55241.849 & 0.516 & -52.3 & -0.2 & 0.3 & 86.9 & 0.0 & 0.01 & Fair \\
\hline
\end{tabular}


Table 4

(Continued)

\begin{tabular}{lcrrrrrrr}
\hline \hline $\begin{array}{c}\text { Heliocentric Julian Date } \\
(\mathrm{HJD}-2,400,000)\end{array}$ & Phase & $\begin{array}{c}V_{1} \\
\left(\mathrm{~km} \mathrm{~s}^{-1}\right)\end{array}$ & $\begin{array}{c}(O-C)_{1} \\
\left(\mathrm{~km} \mathrm{~s}^{-1}\right)\end{array}$ & $\mathrm{Wt}_{1}$ & $\begin{array}{c}V_{2} \\
\left(\mathrm{~km} \mathrm{~s}^{-1}\right)\end{array}$ & $\begin{array}{r}(O-C)_{2} \\
\left(\mathrm{~km} \mathrm{~s}^{-1}\right)\end{array}$ & $\mathrm{Wt}_{2}$ & Source $^{\mathrm{a}}$ \\
\hline 55247.962 & 0.507 & -52.8 & -0.5 & 0.3 & 84.3 & -3.0 & 0.01 & Fair \\
55269.838 & 0.055 & 38.2 & -0.1 & 0.3 & -92.9 & 0.4 & 0.01 & Fair \\
55275.791 & 0.020 & 40.6 & 0.0 & 0.3 & -96.0 & 2.0 & 0.01 & Fair \\
55280.804 & 0.833 & 17.8 & 0.3 & 0.3 & -53.1 & -1.2 & 0.01 & Fair \\
55286.751 & 0.797 & 8.3 & 0.4 & 0.3 & -31.0 & 1.8 & 0.01 & Fair \\
55291.757 & 0.609 & -42.3 & -0.4 & 0.3 & 64.3 & -2.1 & 0.01 & Fair \\
55300.711 & 0.061 & 37.5 & -0.1 & 0.3 & -92.5 & -0.5 & 0.01 & Fair \\
55307.718 & 0.197 & 9.4 & -0.2 & 0.3 & -36.5 & -0.4 & 0.01 & Fair \\
55311.752 & 0.851 & 21.7 & -0.3 & 1.0 & -59.0 & 1.9 & 0.04 & KPNO \\
55312.771 & 0.016 & 41.1 & 0.4 & 1.0 & -99.5 & -1.2 & 0.04 & KPNO \\
55314.753 & 0.338 & -30.0 & 0.1 & 0.3 & 42.9 & -0.1 & 0.01 & Fair \\
55318.809 & 0.995 & 41.8 & 0.8 & 0.3 & -98.9 & -0.2 & 0.01 & Fair \\
55324.667 & 0.945 & 38.9 & 0.6 & 0.3 & -92.6 & 0.7 & 0.01 & Fair \\
55328.698 & 0.599 & -43.2 & 0.4 & 0.3 & 69.9 & -0.1 & 0.01 & Fair \\
55334.677 & 0.568 & -47.8 & 0.3 & 0.3 & 78.9 & 0.0 & 0.01 & Fair \\
55342.682 & 0.866 & 25.6 & 0.1 & 0.3 & -68.1 & -0.2 & 0.01 & Fair \\
55347.681 & 0.677 & -26.8 & -0.5 & 0.3 & 33.6 & -1.9 & 0.01 & Fair \\
55365.645 & 0.590 & -45.1 & 0.0 & 1.0 & 72.3 & -0.6 & 0.04 & KPNO \\
55368.634 & 0.075 & 35.7 & -0.2 & 1.0 & -90.1 & -1.4 & 0.04 & KPNO \\
\hline
\end{tabular}

Notes.

a Fair, Fairborn Observatory; KPNO, Kitt Peak National Observatory.

${ }^{b}$ Low $\mathrm{S} / \mathrm{N}$ spectrum, secondary too weak to be measured.

However, given that its velocities typically come from the measurement of just one or two very weak secondary lines, the $1 \mathrm{~km} \mathrm{~s}^{-1}$ difference between the primary and secondary center-of-mass values is not overly troubling. A simultaneous solution of the appropriately weighted primary and secondary velocities produced a period of 6.167019 days.

From their initial orbital analysis, Worek et al. (1978) concluded that the orbit of 41 Sex is essentially circular. Follow-up work generally validated that result. However, Sreedhar Rao \& Abhyankar (1992) concluded that the very modest eccentricity of 0.034 , which they obtained for their combined solution, is real. Our independent KPNO and Fairborn orbital solutions of the primary produce extremely small eccentricities of $0.0016 \pm$ 0.0017 and $0.0009 \pm 0.016$, respectively. Thus, following the precepts of Lucy \& Sweeney (1971) we have concluded that the orbit is indeed circular.

In principle, the older literature velocities of the primary could improve the precision of the period. However, the vast majority are from photographic plates that have significantly lower resolution than our observations. Nevertheless, we have examined two different older data sets. The 22 KPNO photographic plate velocities of Abt \& Levy (1985) were obtained between 1976 and 1978, extending the time baseline by 25 years. However, from a comparison of orbital solutions the weights of those velocities, relative to our KPNO CCD velocities, are just 0.01 . The resulting combined solution of the two data sets does not significantly change the orbital period. We also examined the six CCD velocities of Worek (1998) to see how they compare with our orbit for the primary. Although they appear to be consistent with our orbit, they have residuals that on average are $0.9 \mathrm{~km} \mathrm{~s}^{-1}$ too positive. This is not overly surprising because such a value is similar to the difference between our systemic velocity and his ( -5.7 and $-4.5 \mathrm{~km} \mathrm{~s}^{-1}$, respectively). Therefore, we have chosen to list in Table 5 the circular orbital solution determined with the new velocities from our two observatories. Figure 4 compares our primary and secondary velocities with the calcu-

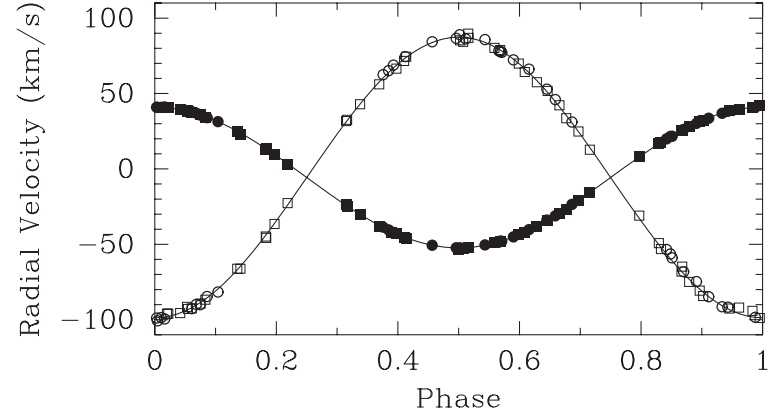

Figure 4. Radial velocities of 41 Sex compared with the computed velocity curves. Filled and open symbols represent the primary and secondary, respectively. Circles, KPNO; squares, Fairborn Observatory. Zero phase is a time of maximum velocity of the primary.

lated velocity curves. For a circular orbit, the element $T$, a time of periastron passage, is undefined. Thus, as recommended by Batten et al. (1989), $T_{0}$, a time of maximum velocity for the primary, is used instead.

In addition to our elements, Table 5 lists those of Abt \& Levy (1985) and Worek (1998). The derived periods and semiamplitudes of the three solutions are in excellent accord. The three values of the systemic velocity are $-6.2,-4.5$, and $-5.7 \mathrm{~km} \mathrm{~s}^{-1}$ according to Abt \& Levy (1985), Worek (1998), and this work. The uncertainties of the values indicate that the difference between the systemic velocity of Abt \& Levy (1985) and our work is slightly more than $1 \sigma$. The $1.2 \mathrm{~km} \mathrm{~s}^{-1}$ difference between the results of Worek (1998) and our work is a $6 \sigma$ result, but nevertheless, perhaps is simply a difference in observatory zero points rather than an indication of a third star in the system. We note that our velocities cover 10 years and are more precise than those previously obtained, yet they show no systematic, long-term velocity change attributable to a third star. 
Table 5

Orbital Elements and Related Parameters of 41 Sex

\begin{tabular}{lccc}
\hline \hline \multicolumn{1}{c}{ Parameter } & Abt \& Levy (1985) & Worek (1998) & This Study \\
\hline$P$ (days) & $6.16663 \pm 0.00002$ & $6.16699 \pm 0.00002$ & $6.1670194 \pm 0.0000070$ \\
$T_{0}(\mathrm{HJD})$ & $2440000.162 \pm 0.012$ & $2444730.060 \pm 0.006$ & $2453690.7442 \pm 0.0011$ \\
$e$ & $0.025 \pm 0.010$ & $0.014 \pm 0.006$ & 0.0 (adopted) \\
$\omega_{1}(\mathrm{deg})$ & $0.0 \pm 0.7$ & $272 \pm 24$ & $\ldots$ \\
$K_{1}\left(\mathrm{~km} \mathrm{~s}^{-1}\right)$ & $46.9 \pm 0.5$ & $46.8 \pm 0.3$ & $46.670 \pm 0.040$ \\
$K_{2}\left(\mathrm{~km} \mathrm{~s}^{-1}\right)$ & $\ldots$ & $\ldots$ & $93.06 \pm 0.20$ \\
$\gamma\left(\mathrm{km} \mathrm{s}^{-1}\right)$ & $-6.2 \pm 0.4$ & $-4.5 \pm 0.2$ & $-5.685 \pm 0.033$ \\
$m_{1} \sin ^{3} i\left(M_{\odot}\right)$ & $\ldots$ & $\ldots$ & $1.1637 \pm 0.0060$ \\
$m_{2} \sin ^{3} i\left(M_{\odot}\right)$ & $\ldots$ & $\ldots .97 \pm 0.03$ & $0.5836 \pm 0.0019$ \\
$a_{1} \sin i\left(10^{6} \mathrm{~km}\right)$ & 3.976 & $\ldots$ & $3.9577 \pm 0.0034$ \\
$a_{2} \sin i\left(10^{6} \mathrm{~km}\right)$ & $\ldots$ & 1.5 & $7.892 \pm 0.017$ \\
rms residual $\left(\mathrm{km} \mathrm{s}{ }^{-1}\right)($ unit weight) & 1.97 & & 0.2 \\
\hline
\end{tabular}

\section{SPECTRAL TYPES AND MAGNITUDE DIFFERENCE}

Strassmeier \& Fekel (1990) identified several luminositysensitive and temperature-sensitive line ratios in the 6430$6465 \AA$ region. They employed those critical line ratios and the general appearance of the spectrum as spectral-type criteria. As discussed below, the abundance peculiarities of the A stars that dominate our systems make normal spectral classification essentially impossible in our limited $84 \AA$ A region. In addition, for stars that are hotter than early-G spectral class, the line ratios in that wavelength region have little sensitivity to luminosity. However, the luminosity class may be determined by computing the absolute visual magnitude with the Hipparcos parallax and comparing that magnitude to evolutionary tracks or a table of canonical values for giants and dwarfs.

Spectra of our two binaries were compared with the spectra of a number of A, F, and early-G stars primarily from the lists of Abt \& Morrell (1995) and Fekel (1997). The reference star spectra were obtained at KPNO with the same telescope, spectrograph, and detector as our binary star spectra. To facilitate a comparison, various combinations of the reference-star spectra were rotationally broadened, shifted in radial velocity, appropriately weighted, and added together with a computer program developed by Huenemoerder \& Barden (1984) and Barden (1985) in an attempt to reproduce the binary spectra.

Analysis of our two star systems has met with very limited success because three of the four components are Am stars and lines of the fourth component are extremely weak. Classical Am stars have spectral classes of A4-F1, determined from their hydrogen lines (Abt \& Morrell 1995). Such stars are noted as having peculiar spectra because lines of their metallic elements such as iron and strontium are stronger than expected compared to the hydrogen classification, while elements such as calcium and scandium are weaker (Abt \& Morrell 1995). There are no hydrogen lines in our limited $6430 \AA$ wavelength region, and the iron and calcium abundance peculiarities vary from star to star, making it impossible to adequately characterize the combined spectrum of the two components with our limited number of reference spectra.

\subsection{HD 434}

Despite being unable to determine spectral classes for the components of HD 434, we have compared the spectrum of this binary with several reference star spectra and have the following comments.

Abt (2004) classified the combined spectrum of HD 434 as an Am star with A2, A6, and F0 spectral classes for its
$\mathrm{Ca} \mathrm{K}$, hydrogen, and metal lines, respectively. We find that in the 6430 A region, the spectrum of the Am star HR 7431, which Abt \& Morrell (1995) classified as A2, A7, and F0, is a reasonable fit to the relative strength of the primary's iron and calcium lines. Thus, we adopt A2 and F0 for the calcium and metal spectral classes of the primary. The iron lines of the secondary are more consistent with those of HR 5075, classified as F2 V by Abt \& Morrell (1995), than with those of HR 7431, but the calcium lines of the secondary are much weaker than those of HR 5075, and so we adopt A2 and F2 for the calcium and metal spectral classes of the secondary. Thus, we confirm the conclusion of Iliev et al. (2001) that both components are Am stars. From our various spectrum fits to the iron lines, we estimate a $V$ mag difference of $0.8 \pm 0.2$. This assumes that the surface abundances of the two stars are the same, which, because both components are Am stars, is not necessarily the case. However, a similar or identical magnitude difference can be estimated from the mass ratio of HD 434 and the canonical properties of A and early-F stars, if one assumes that the spectral type of the primary is somewhat earlier than the composite hydrogen type of Abt (2004) and that the secondary type is somewhat later. For example, in his Table B1, Gray (1992) lists masses for A5 V and F1 V stars of $1.86 M_{\odot}$ and $1.51 M_{\odot}$, respectively. This combination results in a mass ratio of 0.81 , identical to our mass ratio for HD 434 from Table 3. Those two spectral types have corresponding $M_{v}$ magnitudes of 2.0 and 2.8, resulting in a magnitude difference of 0.8 , which is the value that we have estimated from our spectrum fits to the iron lines.

\section{2. $41 \mathrm{Sex}$}

Because the primary of 41 Sex, like both components of HD 434, is an Am star, we are unable to determine its hydrogen line spectral class. The large magnitude difference between the primary and the secondary means that the secondary spectral class is not well determined. Nevertheless, we discuss the results of our comparison of the spectrum of $41 \mathrm{Sex}$ with several reference star spectra.

Abt \& Morrell (1995) classified the combined spectrum of 41 Sex as an Am star with A3, A7, and A9 for its Ca K, hydrogen, and metal lines, respectively. We find that in the $6430 \AA$ A region, the spectrum of the Am star HR 7431, which Abt \& Morrell (1995) classified as A2, A7, and F0 for its Ca K, hydrogen, and metal lines, respectively, is a reasonable fit to the relative strength of the primary's $\mathrm{Fe}$ I and $\mathrm{Fe}$ II lines but its calcium lines are not nearly as strong as those of 41 Sex. From a comparison with several other reference stars we adopt A5 and F0 for the primary's calcium and metal spectral classes, respectively. The 
very weak spectrum of the secondary is consistent with that of a late-F or an early-G star.

\section{BASIC PROPERTIES}

\section{1. $H D 434$}

For HD 434 we begin by adopting a $V$ mag of 6.47 and a $B-V$ color of $0.242 \mathrm{mag}$ from the Hipparcos catalog (Perryman et al. 1997). With our magnitude difference of 0.8 , the individual $V$ magnitudes are 6.89 and 7.69 for the primary and secondary, respectively. The new Hipparcos parallax reduction by van Leeuwen (2007) produces a value of $9.02 \pm 0.49$ mas and corresponds to a distance of $110.9 \pm 6.0 \mathrm{pc}$. Even though there may be a slight amount of reddening at this distance, we assume that it is negligible. The resulting absolute magnitudes are $M_{V}=1.7 \pm 0.2 \mathrm{mag}$ and $M_{V}=2.5 \pm 0.2 \mathrm{mag}$ for the primary and secondary, respectively. From Johnson (1966) we assume $B-V$ colors of 0.17 for the primary and 0.36 for the secondary, which, when combined for the two stars, produce a $B-V$ color similar to the observed value. Then, from Table 3 of Flower (1996), we obtain the bolometric corrections and effective temperatures of the two components. Finally, with the use of the Stefan-Boltzmann law, the luminosities of the primary and secondary are $L_{1}=16.7 \pm 3.6 L_{\odot}$ and $L_{2}=8.0 \pm 1.7 L_{\odot}$, respectively, while the radii are $R_{1}=$ $2.2 \pm 0.3 R_{\odot}$ and $R_{2}=2.0 \pm 0.3 R_{\odot}$, respectively. The uncertainties in the computed quantities are dominated by the parallax uncertainty, the magnitude difference uncertainty, and the effective temperature uncertainty with the latter estimated to be $\pm 300 \mathrm{~K}$.

\section{2. $41 \operatorname{Sex}$}

Stockton \& Fekel (1992) concluded that binaries with mass ratios as small as 0.6 and magnitude differences somewhat greater than 2.5 mag can be detected at $6430 \AA$. With a smaller mass ratio of 0.5 , the components of 41 Sex have an even greater magnitude difference. Because of the faintness of the secondary, that component will have only a very slight effect on the $V$ mag and color of the primary. For example, if the $\Delta V$ mag between the primary and secondary is assumed to be $3.0 \mathrm{mag}$, then the $V$ mag of the primary is 5.87, while increasing the magnitude difference to 3.5 makes the $V$ mag of the primary 5.84 . As discussed below, in the following computations we adopt $\Delta V=3.2 \mathrm{mag}$, which with a $V$ mag of 5.80 for the combined system from Hipparcos (Perryman et al. 1997) results in $V=$ $5.86 \mathrm{mag}$ for the primary. The new reduction of the Hipparcos parallax produces a value of $10.50 \pm 0.34$ mas (van Leeuwen 2007), which corresponds to a distance of $95.2 \pm 3.1 \mathrm{pc}$. At such a distance we assume that the system is not significantly reddened. Our assumed magnitude difference and the parallax result in an absolute magnitude of $M_{v}=0.97 \pm 0.12$ mag for the primary. From the Hipparcos catalog (Perryman et al. 1997), the $B-V$ color of the 41 Sex system is 0.156 . Given the very modest effect of the secondary, we adopt a slightly smaller value of 0.15 for the primary. The bolometric correction and effective temperature come from Table 3 of Flower (1996). Then from the Stefan-Boltzmann law the luminosity of the primary, $L_{1}$, is $31.9 \pm 3.6 L_{\odot}$, while the radius is $R_{1}=2.9 \pm 0.2 R_{\odot}$.

The properties of the secondary are much less secure. We compare the $M_{v}$ of the primary and the components' mass ratio with the canonical stellar properties listed in Table B1 of Gray (1992) and conclude that the secondary is most probably a late-F or early-G star, which is in agreement with our rough classification of this star. From that table we estimate a $V$ mag difference of $3.2 \pm 0.3$. This leads to $L_{2}=1.8 \pm 0.5 L_{\odot}$ and $R_{2}=1.3 \pm 0.2 R_{\odot}$. The uncertainties in the computed quantities of the primary and secondary are dominated by the parallax and the effective temperature uncertainties as well as the magnitude difference for the secondary. The temperature uncertainty is estimated to be $\pm 200 \mathrm{~K}$ for the primary and secondary.

\section{CIRCULARIZATION AND SYNCHRONIZATION}

The two main theories of orbital circularization and rotational synchronization (see Zahn 1977; Tassoul \& Tassoul 1992) disagree significantly on absolute time scales but do agree that synchronization should occur first. Observationally, Matthews \& Mathieu (1992) examined 62 spectroscopic binaries with A-type primaries and periods less than 100 days. They concluded that all systems with orbital periods $\lesssim 3$ days have circular or nearly circular orbits. They also found that many binaries with periods in the range of 3-10 days have circular orbits. With a period of 34.26 days it is not surprising that HD 434 still has an eccentric orbit. Also unsurprising is the circular orbit of $41 \mathrm{Sex}$, which has a period of only 6.167 days.

In an eccentric orbit, Hut (1981) has shown that the rotational angular velocity of a star will tend to synchronize with that of the orbital motion at periastron. For HD 434 we compute the pseudosynchronous period with Equation (42) of Hut (1981).

To help in assessing pseudosynchronization and synchronization in our two binaries, we have determined projected rotational velocities from our red-wavelength KPNO spectra with the procedure of Fekel (1997). For A-type stars the measured line broadening was converted to a $v \sin i$ value. For stars with later spectral classes, macroturbulent broadening has been taken into account. Following Fekel (1997), for late-F and early-G stars a value of $3 \mathrm{~km} \mathrm{~s}^{-1}$ was used. To convert the $v \sin i$ values into equatorial rotational velocities, we assume, as is generally done, that the axes of the orbital and rotational planes are parallel, so the inclinations are equal.

\section{1. $H D 434$}

To determine whether the components of HD 434 are rotating pseudosynchronously, we compare our observed velocities with the predicted pseudosynchronous velocities. For HD 434 our $v \sin i$ values, averaged from two spectra, are $33 \pm 2.0$ and $30 \pm 3.0 \mathrm{~km} \mathrm{~s}^{-1}$ for the primary and secondary, respectively. These values are in good agreement with those of Iliev et al. (2001), who estimated projected rotational velocities of about 32 and $27 \mathrm{~km} \mathrm{~s}^{-1}$ for the two stars. The minimum masses of the two components are relatively small; both are less than $1 M_{\odot}$. From our comparison with the solar-abundance evolutionary tracks of Girardi et al. (2000) we adopt a mass of $1.9 M_{\odot}$ for the primary (Figure 5). Then that mass, combined with its $m$ $\sin ^{3} i$ value (Table 3), results in an orbital inclination of $54^{\circ}$. If the rotational inclination is the same as that of the orbit, then the observed equatorial rotational velocities are 40 and $33 \mathrm{~km} \mathrm{~s}^{-1}$ for the primary and secondary, respectively. The pseudosynchronous rotation period of HD 434 is 20.9 days. This value plus our computed radii from the Stefan-Boltzmann law produces pseudosynchronous rotational velocities of $5 \mathrm{~km} \mathrm{~s}^{-1}$ for both the primary and secondary. Thus, the two components of HD 434 are rotating 6-8 times more rapidly than their pseudosynchronous values. 


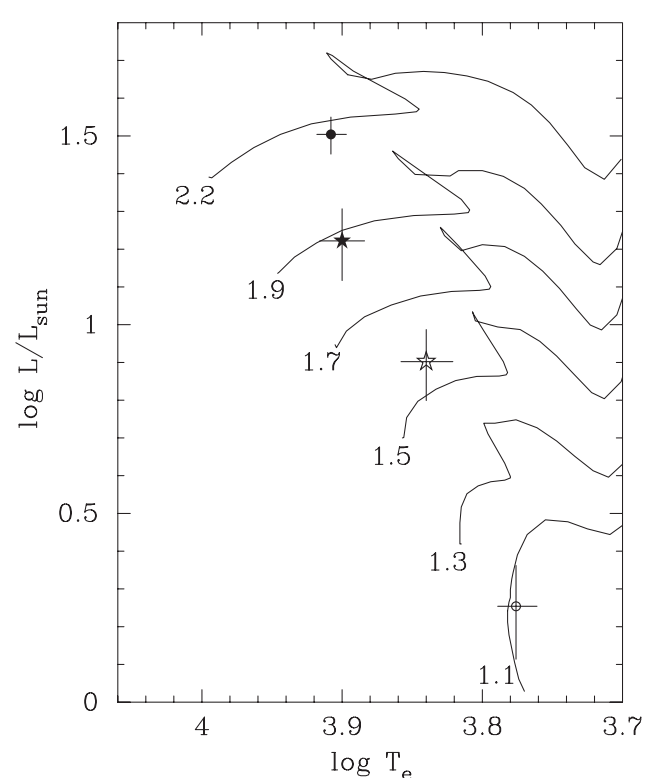

Figure 5. Positions of the components of HD 434 (stars) and 41 Sex (circles) are compared with the $1.1,1.3,1.5,1.7,1.9$, and $2.2 M_{\odot}$ solar-abundance evolutionary tracks of Girardi et al. (2000) in a theoretical H-R diagram. The more massive component in each system corresponds to the filled symbol. Our estimated uncertainties are shown.

\section{2. $41 \mathrm{Sex}$}

To determine whether the components of 41 Sex are synchronously rotating, we compute equatorial velocities from our projected rotational velocities and then compare them with the predicted pseudosynchronous velocities. For 41 Sex our $v \sin i$ values, averaged from 35 spectra for the primary and 29 spectra for the secondary, are $20 \pm 1$ and $7 \pm 3 \mathrm{~km} \mathrm{~s}^{-1}$, respectively. Fekel (2003) obtained a $v \sin i$ value of $21 \mathrm{~km} \mathrm{~s}^{-1}$ from a small subset of our spectra. The minimum masses of the two components (Table 5) are relatively small compared to the expected masses for their spectral types (Gray 1992). Thus, we compare the position of the primary in the H-R diagram with the solar abundance evolutionary tracks of Girardi et al. (2000), which produces a mass estimate of $2.18 M_{\odot}$. That value, combined with the $m \sin ^{3} i$ value of the primary, results in an orbital inclination of $54^{\circ}$, which by chance is the same value that we have estimated for HD 434. If the axes of the rotational and orbital inclinations are parallel, then the observed equatorial rotational velocities are 25 and $9 \mathrm{~km} \mathrm{~s}^{-1}$. The synchronous rotational velocities for the primary and secondary, computed with our radii from the Stefan-Boltzmann law, are 24 and $10 \mathrm{~km} \mathrm{~s}^{-1}$, respectively. Thus, we conclude that both the primary and secondary are rotating synchronously. This result accords with theory, since the binary orbit is already circularized.

\section{LINE DEPTHS OF 41 SEX}

From 66 and $33 \AA \mathrm{mm}^{-1}$ photographic spectra, Sreedhar Rao et al. (1990) presented evidence that the primary of 41 Sex has phase-modulated line depth variations. They stated that many blended metallic lines in their blue wavelength spectra have depth changes of 10\%-20\% and appear to systematically peak in strength around orbital phases $0.25,0.50,0.75$, and 1.0 , relative to the time of maximum positive velocity. They argued that these line depth changes were similar to those seen in various metal lines of Ap stars and so likely had a similar explanation. The hallmark of this latter class of peculiar stars

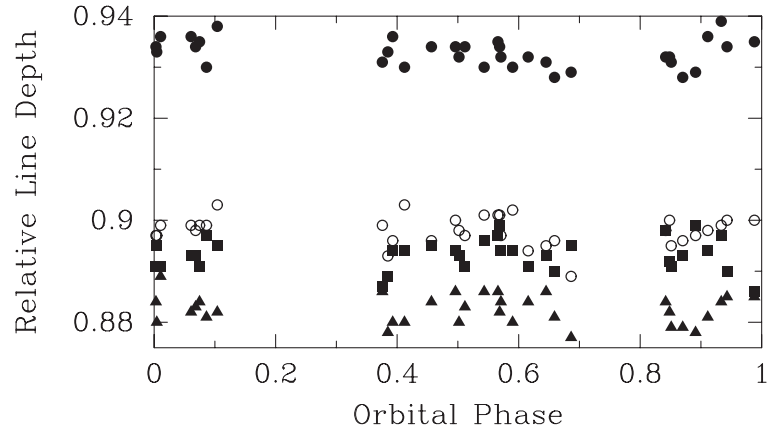

Figure 6. Relative line depths vs. orbital phase for four lines of the $41 \mathrm{Sex}$ primary. Zero phase is a time of maximum positive velocity. Solid circles: Fe I at $6393.612 \AA$, open circles: Fe I at $6400.009 \AA$, squares: Fe II at $6416.928 \AA$, and triangles: Ca I at $6439.083 \AA$.

Table 6

Line Depths of 41 Sex

\begin{tabular}{lccc}
\hline \hline Ion & $\begin{array}{c}\text { Wavelength } \\
(\AA)\end{array}$ & $\begin{array}{c}\text { Mean } \\
\text { Line Depth }\end{array}$ & Sigma \\
\hline $\mathrm{Fe} \mathrm{I}_{\mathrm{I}}$ & 6393.612 & 0.933 & 0.003 \\
$\mathrm{Fe} I$ & 6400.009 & 0.898 & 0.003 \\
$\mathrm{Fe}$ II & 6416.928 & 0.893 & 0.003 \\
$\mathrm{Ca} I$ & 6439.083 & 0.883 & 0.003 \\
\hline
\end{tabular}

is that they have much stronger than normal absorption lines of rare earth elements (Lueftinger et al. 2003), produced by very strong magnetic fields that have caused those particular elements to coalesce into localized surface regions. As an Ap star rotates, periodic changes in the strength of these lines are seen. The technique of Doppler imaging provides surface maps of the changing distributions (e.g., Lueftinger et al. 2003).

As a result of their analysis, Sreedhar Rao et al. (1990) concluded that the Am star primary of 41 Sex has characteristics that link the Am and Ap groups. However, they did caution that higher resolution CCD spectra would be useful to confirm the spectral line variations. Worek's ongoing interest in the 41 Sex system led him to obtain such observations. His careful analysis of new blue wavelength spectra (Worek 1998) did not confirm the results of Sreedhar Rao et al. (1990), and he suggested alternative possible reasons for the line variability claimed by Sreedhar Rao et al. (1990).

We analyzed 34 red wavelength KPNO spectra to examine further the possible line depth changes in the primary of 41 Sex. Our spectra typically have signal-to-noise ratios of 200-300. Although the red region of the spectrum is far less crowded than the blue region, the metal lines are generally much weaker than those at blue wavelengths. Table 6 lists the two Fe I lines, one Fe II line, and one Ca I line that we measured. The spectra were normalized to unity and the continuum rectified. A Gaussian curve was fitted to each observed line profile. The depths (one minus the residual intensity) of the four lines are plotted versus the orbital phase in Figure 6, and the average depth and standard deviation are listed in Table 6 . The orbital phases are those given in Table 4, where the zero phase is a time of maximum velocity of the primary. Despite gaps in our phase coverage near orbital conjunctions at phases 0.25 and 0.75 , we find no line variations at the relative intensity level of $0.01-0.02$ suggested by the results of Sreedhar Rao et al. (1990). The depths of all four lines have $\sigma=0.003$ (Table 6), and for three of the four lines, $82 \%$ of the line depths are within $1 \sigma$ of the average values. The exception is the $\mathrm{Fe}$ II line, which has $74 \%$ of its measures within 
$1 \sigma$. Like Worek (1998), we are unable to confirm the results of Sreedhar Rao et al. (1990).

\section{CONCLUSIONS}

We have detected the secondary component in two previously known spectroscopic binaries, HD 434 and 41 Sex, and for the first time determined double-lined orbits for them. Because the secondary lines of HD 434 were previously unresolved, several of our orbital elements are substantially different from earlier solutions. With the detection of the faint secondary component of $41 \mathrm{Sex}$ we have enhanced the utility of that system for the determination of its basic properties.

We have confirmed the conclusion of Iliev et al. (2001) that both components of HD 434 are Am stars. The primary of 41 Sex is also an Am star, while its secondary component is a late-F or early-G dwarf. Comparison with evolutionary tracks indicates that all four stars are still on the main sequence.

The components of HD 434 are rotating 6-8 times faster than their predicted pseudosynchronous velocities. On the other hand, the components of 41 Sex are rotating synchronously.

From the Hipparcos parallaxes and adopted masses of the two systems we have estimated the maximum angular separations of the components. The orbit of HD 434 is eccentric, and so we determined its maximum nodal separation (e.g., McAlister 1976; Halbwachs 1981) of 3.3 mas. The orbit of 41 Sex is circular, enabling us, like Halbwachs (1981), to use Kepler's third law to estimate an angular semimajor axis of 1.0 mas. Although these separations are small, they are within the scope of modern optical interferometers. Thus, when our spectroscopic results are complemented with high-quality interferometric results, accurate three-dimensional orbits, masses, and distances for the systems will follow.

For the primary in 41 Sex we measured the line depths of four metal lines. Like Worek (1998), we are unable to confirm the 10\%-20\% line depth variations claimed by Sreedhar Rao et al. (1990).

We thank J. Tomkin for providing us with his velocities from the $2.1 \mathrm{~m}$ telescope at McDonald Observatory. The help of Daryl Willmarth in support of the KPNO coudé feed observations is appreciated. The research at Tennessee State University was supported in part by NASA grant NCC5-511 and NSF grant HRD-9706268.

\section{REFERENCES}

Abt, H. A. 1961, ApJS, 6, 37

Abt, H. A. 2004, ApJS, 155, 175

Abt, H. A., \& Levy, S. G. 1985, ApJS, 59, 229

Abt, H. A., \& Morrell, N. I. 1995, ApJS, 99, 135

Barden, S. C. 1985, ApJ, 295, 162

Barker, E. S., Evans, D. S., \& Laing, J. D. 1967, R. Obs. Bull., 130, 355
Batten, A. H., Fletcher, J. M., \& MacCarthy, D. G. 1989, Publ. Dom. Astrophys. Obs., 17, 1

Bertaud, Ch. 1970, A\&AS, 1, 7

Boden, A. F., Torres, G., \& Latham, D. W. 2006, ApJ, 644, 1193

Budaj, J., Iliev, I. Kh., Barzova, I. S., Ziznovsky, J., Zverko, J., \& Stateva, I. 2003, IBVS, 5423, 1

Cowley, A., Cowley, C., Jaschek, M., \& Jaschek, C. 1969, AJ, 74, 375

Cunha, M. S. 2007, A\&AR, 14, 217

Eaton, J. A., \& Williamson, M. H. 2004, Proc. SPIE, 5496, 710

Eaton, J. A., \& Williamson, M. H. 2007, PASP, 119, 886

Fekel, F. C. 1997, PASP, 109, 514

Fekel, F. C. 2003, PASP, 115,807

Fekel, F. C., Boden, A. F., Tomkin, J., \& Torres, G. 2009a, ApJ, 695, 1527

Fekel, F. C., Tomkin, J., \& Williamson, M. H. 2009b, AJ, 137, 3900

Fekel, F. C., Tomkin, J., \& Williamson, M. H. 2010, AJ, 139, 1579

Flower, P. J. 1996, ApJ, 469, 355

Girardi, L., Bressan, A., Bertelli, G., \& Chiosi, C. 2000, A\&AS, 141, 371

Gray, D. F. 1992, The Observation and Analysis of Stellar Photospheres (Cambridge: Cambridge Univ. Press)

Halbwachs, J. L. 1981, A\&AS, 44, 47

Hoffleit, D. 1982, The Bright Star Catalogue (4th rev. ed.; New Haven, CT: Yale Univ. Observatory)

Hube, D. P., \& Gulliver, A. F. 1985, J. R. Astron. Soc. Canada, 79, 49

Huenemoerder, D. P., \& Barden, S. C. 1984, BAAS, 16, 510

Hummel, C. A., et al. 2001, AJ, 121, 1623

Hut, P. 1981, A\&A, 99, 126

Iliev, I. Kh., Budaj, J., Ziznovsky, J., \& Zverko, J. 2001, IBVS, 5051, 1

Johnson, H. L. 1966, ARA\&A, 4, 193

Lucy, L. B., \& Sweeney, M. A. 1971, AJ, 76, 544

Lueftinger, T., Kuschnig, R., Piskunov, N. E., \& Weiss, W. W. 2003, A\&A, 406 1033

Margoni, R., Munari, U., \& Stagni, R. 1992, A\&AS, 93, 545

Matthews, L. D., \& Mathieu, R. D. 1992, in ASP Conf. Ser. 32, Complimentary Approaches to Double and Multiple Star Research, IAU Colloq. 135, ed. H. A. McAlister \& W. I. Hartkopf (San Francisco, CA: ASP), 244

McAlister, H. A. 1976, PASP, 88, 317

McCarthy, J. A., Sandiford, B. A., Boyd, D., \& Booth, J. 1993, PASP, 105, 881

Palmer, D. R., Walker, E. N., Jones, D. H. P., \& Wallis, R. E. 1968, R. Greenwich Obs. Bull., 135, 385

Perryman, M. A. C., et al. 1997, A\&A, 323, L49

Pourbaix, D., et al. 2004, A\&A, 424, 727

Quirrenbach, A. 2001, ARA\&A, 39, 353

Royer, F., Grenier, S., Baylac, M.-O., Gomez, A. E., \& Zorec, J. 2002, A\&A, 393, 897

Scarfe, C. D., Batten, A. H., \& Fletcher, J. M. 1990, Publ. Dom. Astrophys. Obs., 18,21

Shajn, G. A. 1951, Izv. Krymsk Astrofiz. Obs., 7, 124

Sreedhar Rao, S., \& Abhyankar, K. D. 1992, MNRAS, 258, 819

Sreedhar Rao, S., Abhyankar, K. D., \& Nagar, P. 1990, ApJ, 365, 336

Stockton, R. A., \& Fekel, F. C. 1990, MNRAS, 256, 575

Strassmeier, K. G., \& Fekel, F. C. 1990, A\&A, 230, 389

Tassoul, J.-L., \& Tassoul, M. 1992, ApJ, 395, 259

Tomkin, J., \& Fekel, F. C. 2006, AJ, 131, 2652

Tomkin, J., \& Fekel, F. C. 2008, AJ, 135, 555

Torres, G., Andersen, J., \& Giménez, A. 2010, A\&AR, 18, 67

van Leeuwen, F. 2007, A\&A, 474, 653

Walker, N. 1966, Observatory, 86, 154

Wolfe, R. H., Horak, H. G., \& Storer, N. W. 1967, in Modern Astrophysics, ed. M. Hack (New York: Gordon \& Breach), 251

Worek, T. F. 1998, PASP, 110, 580

Worek, T. F., Beardsley, W. R., \& King, M. W. 1978, AJ, 83, 303

Worek, T. F., Zizka, R., King, M. W., \& Beardsley, W. R. 1986, PASP, 98, 238 Zahn, J.-P. 1977, A\&A, 57, 383 